\title{
The effect of ivermectin on non-severe and severe COVID-19 disease and gender-based difference of its effectiveness
}

\author{
Syed Muhammad Zubair, Muhammad Waleed Chaudhry, Ali Bin Sarwar Zubairi, Talha Shahzad, \\ Aqusa Zahid, Ibrahim Ali Khan, Javaid Ahmed Khan, Muhammad Irfan \\ Section of Pulmonary Medicine, Department of Medicine Aga Khan University Hospital, Karachi, Pakistan
}

\begin{abstract}
The COVID-19 pandemic has led to mortality and morbidity since December 2019. Many possible treatment options have
\end{abstract}

Correspondence: Muhammad Irfan, Professor, Section of Pulmonary Medicine, Department of Medicine, Aga Khan University Hospital,

National Stadium Road, Karachi, Sindh 74800, Pakistan.

Tel. +92.021.34864664 - Mobile: +92.3002111459.

E-mail: muhammad.irfan@aku.edu

Key words: Ivermectin; COVID-19; SARS-CoV-2; virus; treatment; therapy.

Contributions: SMZ, MWC, data collection, statistical analysis, literature review, manuscript writing; ABSZ, manuscript writing, reviewing, editing; TS, IAK, JAK, manuscript reviewing for intellectual content, editing; AZ, data collection, manuscript reviewing for intellectual content, editing; MI, study concept, manuscript writing, reviewing, editing. All the authors read and approved the final version of the manuscript and agreed to be accountable for all aspects of the work.

Ethics approval: The study protocol was approved by the Ethics Review Committee (ERC) of the Aga Khan University Hospital (application no: 2021-5902-16610).

Conflict of interest: The authors declare that they have no competing interests, and all authors confirm accuracy.

Conference presentation: The abstract of this article has been presented as an e-poster at the at virtual European Respiratory Society Congress 2021 on 07-09-2021 with the title "Role of ivermectin in hospitalized patients with mild to moderate COVID-19".

Received for publication: 25 August 2021.

Accepted for publication: 13 January 2022.

Publisher's note: All claims expressed in this article are solely those of the authors and do not necessarily represent those of their affiliated organizations, or those of the publisher, the editors and the reviewers. Any product that may be evaluated in this article or claim that may be made by its manufacturer is not guaranteed or endorsed by the publisher.

${ }^{\circ}$ Copyright: the Author(s), 2022

Licensee PAGEPress, Italy

Monaldi Archives for Chest Disease 2022; $92: 2062$

doi: 10.4081 /monaldi.2022.2062

This article is distributed under the terms of the Creative Commons Attribution-NonCommercial International License (CC BY-NC 4.0) which permits any noncommercial use, distribution, and reproduction in any medium, provided the original author(s) and source are credited. been advised till date. The role of ivermectin in the treatment of COVID-19 disease remains controversial. The aim of our study was to evaluate the effect of ivermectin in hospitalized patients with non-severe and severe COVID-19 disease. We conducted a retrospective cohort study that compared outcomes in 2 groups of COVID-19 patients hospitalized at the largest tertiary care center of Pakistan. The study group was given ivermectin along with standard treatment of covid-19 disease; the comparison group was not. Data on mortality, inflammatory markers such as C-reactive protein (CRP) and ferritin, length of hospital stay and baseline characteristics were collected from Aga Khan University's database from October 2020 till February 2021. Statistical analysis was done to determine the effectiveness of ivermectin in non-severe and severe COVID-19. Comparison of effectiveness of ivermectin in both genders was also conducted. The cohort included 188 patients out of which 90 were treated with ivermectin. Mortality and length of hospitalization was not found to be significantly different in the study group compared with the control group (5.6\% vs $5.1 \% ; \mathrm{p}=0.87$ and 5 days $v s 4$ days; $\mathrm{p}=0.27$ ). Analysis of secondary outcomes did not yield statistically significant results, apart from ferritin levels which were significantly less in patients treated with ivermectin $(547.1 \mathrm{vs}$ 756.7; $\mathrm{p}=0.03$ ). The ferritin and CRP levels in affected males were higher than in females on admission and discharge. Our findings suggest ivermectin does not significantly affect allcause mortality, length of hospitalization and CRP levels in hospitalized COVID-19 patients. Large scale randomized controlled trials (RCTs) are required to further evaluate the role of ivermectin in covid-19 disease.

\section{Introduction}

The SARS-CoV-2 pandemic that we are currently going through, started in the city of Wuhan in China towards the end of 2019. The pandemic since then has continued the affect the lives of the people throughout the globe. A total of over 162 million people have been infected whereas over 3.3 million lives were claimed by COVID-19 as of 16 May, 2021 [1]. In Pakistan alone 900,552 cases have been confirmed while over 20,000 people passed away due to COVID-19 [2].

Many medications have been proposed as probable treatment but the scientific evidence does not seem to support the use of majority of them. This includes interferon, hydroxychloroquine, chloroquine, conventional anti-virals, mono-clonal antibody, convalescent plasma therapy, and antirheumatics like tocilizumab [36]. Treatment, is largely based on symptomatic management of the patient and prevention of complications. Steroids and remdesivir 
have shown positive outcomes such as decreased mortality or recovery time in patients with COVID-19 [7,8].

Ivermectin seems to be a promising drug for COVID-19. Its ability to impede protein transport into host nucleus via inhibition of importin (IMP) $\alpha / \beta$ receptor are behind its antiviral properties. It can therefore inhibit the replication processes in various RNA and DNA viruses, e.g., influenza, Zika virus, Dengue virus, Porcine circovirus and others $[9,10]$. Another possible contributing mechanism is allosteric modulation of the P2X4 receptor, which leads to the secretion of a chemotactic-chemokine ligand 5 (CCL5) [11]. Some studies suggest that ivermectin might have immunosuppressive effects [12]. This is particularly important since it indicates mitigation of inflammatory response during SARS by the drug. Indeed in-vitro models support the theory that in SARSCoV-2 a similar inhibitory effect leading to decreased replication will be seen [13]. This has spurred clinicians to consider using ivermectin as treatment in COVID-19. Peru has even restarted to prescribe ivermectin to their out-patients - even with arguably insufficient evidence [14]. To the best of our knowledge, genderbased role of ivermectin in COVID-19 has not been discussed in literature as yet. The aim of our study was to evaluate the effect of ivermectin on non-severe and severe COVID-19 disease and to see gender-based difference of its effectiveness.

\section{Materials and Methods}

A retrospective cohort study was conducted on hospitalized patients with COVID-19 disease confirmed by nasopharyngeal RTPCR at the Aga Khan University Hospital (AKUH) located in largest cosmopolitan city of Karachi, in Pakistan between Oct 2020 and Feb 2021. The study protocol was approved by the Ethics Review Committee (ERC) of the AKUH.

AKUH is a tertiary care hospital in Karachi in the largest city of Pakistan. We have recruited non-severe and severe hospitalized patients of COVID-19 disease during study period and divided them in a study group; who received two doses of $12 \mathrm{mg}$ ivermectin $24 \mathrm{~h}$ apart and control group; who had not received ivermectin. Both groups had received other standard treatment according to hospital protocol for management of COVID-19 disease. We have excluded critical COVID-19 patients and patients who were discharged from Emergency Department or those seen in outpatient clinics.

The disease severity was classified according to the WHO classification into non-severe, severe and critically ill patients. Severe COVID-19 was defined as patients having respiratory distress with a respiratory rate of 30 breaths per minute or more and oxygen saturations below $90 \%$ on room air whereas non-severe COVID-19 was defined as absence of signs of severe COVID-19 [15]. Along with the analysis of effect of ivermectin on the study and comparison group, we also analyzed the effect of ivermectin on both the genders to see if there is a gender difference of its effectiveness or not, as this has not been seen and analyzed in literature previously. Our primary outcome was to investigate the effect of ivermectin on all-cause mortality and length of hospitalization in said patients while inflammatory marker levels (CRP and ferritin) at discharge \& differences in the two genders were considered as secondary outcomes.

The data was collected from AKU's Health Information Management Services (HIMS) department on a predesigned questionnaire. Data on age, disease severity (severe or nonsevere), comorbidities, requirement of oxygen post-discharge and exposure to steroids (dexamethasone or prednisone) and/or remdesivir were noted.

\section{Statistical analysis}

All statistical analysis was done using the SPSS (Release 19.0, standard version). A descriptive analysis was performed for demographic features presented as mean \pm SD for quantitative variable like age, gender and comorbidities. Disease severity, inflammatory markers and length of hospital stay were compared between both the groups using Mann-Whitney $U$ test and chisquare test where necessary. Odds ratios with $95 \%$ confidence interval were computed. In all analyses, p-value $<0.05$ was considered statistically significant.

\section{Results}

A total of 188 subjects of non-severe and severe COVID-19 disease were included. Out of 188 patients, 90 patients were a part of the study group whereas the remaining 98 were in the control group. The baseline characteristics of both groups are summarized in Table 1.

There was a male predominance in both groups $(68.9 \%$ in study and $65.3 \%$ in control group). There was no significant difference of age and other comorbidities in both groups. Overall, $61.7 \%$ had severe disease and patients in study group had a slightly more severe disease than the control group ( $65.6 \%$ vs $58.2 \%$; $\mathrm{p}=0.37)$. Likewise, steroids and remdesivir were used more in the study group $(66.7 \%$ vs $48 \%$ ). There was no significant difference noted on CRP levels at discharge $(20.4 \mathrm{mg} / \mathrm{L}$ (interquartile range, $10.3-36 \mathrm{mg} / \mathrm{L}$ ) in study group while $18.5 \mathrm{mg} / \mathrm{L}$ (interquartile range, $8.5-47.5 \mathrm{mg} / \mathrm{L}$ ) in the control group $(\mathrm{p}=0.96)$. We noted an increase in ferritin as the treatment went on across both groups, however the increase in the ivermectin exposed group was significantly less than that in the control group. Median ferritin levels at discharge in the study group was $547.1 \mathrm{mg} / \mathrm{L}$ (interquartile range, $247.5-868.1 \mathrm{mg} / \mathrm{L}$ ) while 756.7 $\mathrm{mg} / \mathrm{L}$ (interquartile range, $313.2-1370.6 \mathrm{mg} / \mathrm{L}$ ) in the control group $(\mathrm{p}=0.03)$ (Table 2). There were 5 mortalities in each group $(5.6 \% \mathrm{vs}$ $5.1 \% ; \mathrm{p}=0.87)$. Age $[\mathrm{OR}=1.15(95 \% \mathrm{CI}, 1.06$ to 1.25$) ; \mathrm{p}<0.001]$ and disease severity $[\mathrm{OR}=5.93$ (95\% CI 0.74-48.17; $\mathrm{p}=0.048)$ was found to be an independent factor of mortality in both groups. Mortality in the two genders was not found to be significantly different [odds ratio $=1.16(95 \% \mathrm{CI}, 0.25$ to 7.17$) ; \mathrm{p}=1]$ (Table 3$)$.

The median length of stay of study group was 5 days (interquartile range, 3-6 days) whereas it was 4 days (interquartile range, 3-6 days) in the control group $(p=0.27)$ (Table 2). Length of hospitalization in females was found to be 4 days (interquartile range, 1-10 days) and in males 4 days (interquartile range, 1-18 days) as well $(\mathrm{p}=0.40)$ (Table 3$)$.

It was also seen that ferritin and CRP levels in affected males were higher than in females at both admission and discharge. Analysis did reveal a significant difference in ferritin on admission $(\mathrm{p}<0.001)$, ferritin on discharge $(\mathrm{p}<0.001)$ and CRP on admission $(p=0.021)$ between the two genders. The difference in CRP on discharge was insignificant $(\mathrm{p}=0.866$ ) (Table 3 and Figure 1). No side effects of ivermectin were seen in the study group.

\section{Discussion}

The role of ivermectin in the treatment of COVID-19 still remains controversial with some studies showing favorable results 
$[16,17]$ while others opposing its use [18]. Our study did not show any statistically significant changes in outcomes like patient mortality, length of hospital stay and differences in CRP levels.

Earlier a study by Caly et al. sparked interest in health professionals across the whole world showing the antiviral properties of ivermectin [13]. This compelled researchers to conduct studies exploring the potential of the drug. There was an influx of studies earlier that claimed ivermectin as an effective option against the pathogen $[16,17,19-23]$. Concerns regarding power of these tests, biases and designs have existed, demanding more solid evidence for the use of ivermectin in COVID-19 - this study opposes that claim.

These findings are also corroborated by a randomized clinical trial which failed to find a decreased time of resolution of symptoms in said patients against placebo [18]. As for some studies which show a positive impact of ivermectin in the disease, many studies have limitations as summarized by National Institutes of Health (NIH) [24].

We agree with Camprubi et al. [25] about the dosage of ivermectin which might be a vital factor. However, its toxicity is a plausible concern, even though studies has shown safety of ivermectin. [26-28]. In the study by Caly et al. [13] the doses used are ten-fold greater than those approved by FDA. This concern, along with noted variable results upon reviewing studies, trials and analyses exploring the use of ivermectin in COVID-19, made the European Medicines Agency advised against its use in COVID-19 [29]. Similarly the COVID-19 Treatment Guidelines Panel at NIH seems to be hesitant, and rightly so, to suggest ivermectin as a treatment option in the disease. In fact recently, they released a statement putting forward concerns related to the quality of studies and trials [30].

A meta-analysis of 3 observational studies by Padhy et al.

Table 1. Characteristics of study and control group.

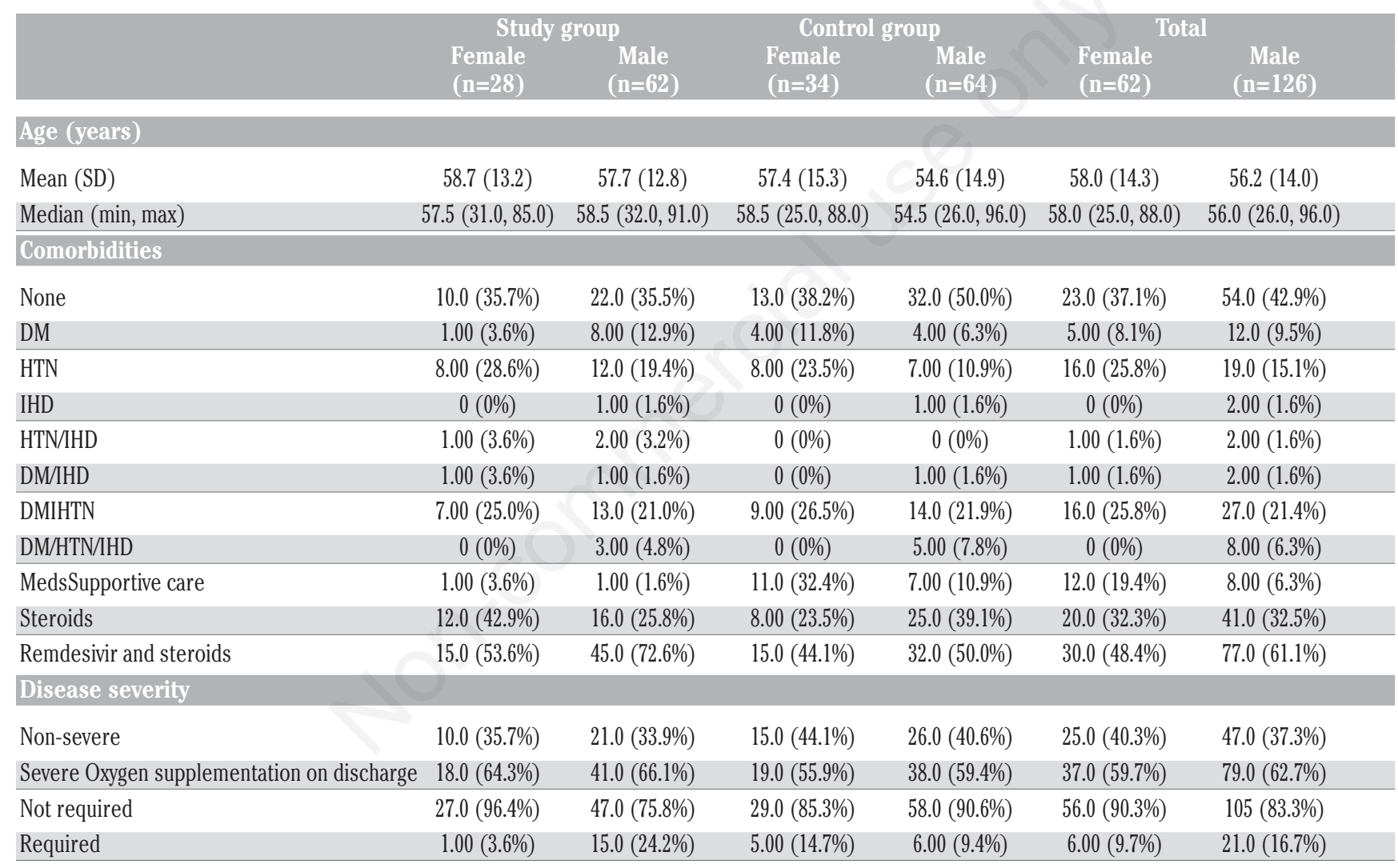

DM, diabetes mellitus; HTN, hypertension; IHD, ischemic heart disease.

Table 2. CRP, ferritin levels, length of hospitalization and outcome in both study and control group.

\begin{tabular}{lcccc} 
& Total & Study group; $\mathrm{n=90}$ & Control group; n=98 & p-value \\
Ferritin on admission & $465.2(233.8-1009.7)$ & $464.8(216.9-846.9)$ & $465.6(256.1-1140.5)$ & $0.58^{*}$ \\
Ferritin on discharge & $640.9(271.1-1160.6)$ & $547.1(247.5-868.1)$ & $756.7(313.2-1370.6)$ & $0.03^{*}$ \\
\hline CRP on admission & $63.1(28.4-122.9)$ & $67.6(35.5-130.5)$ & $58.6(24-119.2)$ & $0.21^{*}$ \\
CRP on discharge & $19(9.2-40)$ & $20.4(10.3-36)$ & $18.5(8.5-47.5)$ & $0.96^{*}$ \\
\hline Length of hospital stay & $4(3-6)$ & $5(3-6)$ & $4(3-6)$ & $0.27^{*}$ \\
OutcomeDischargedMortality & $179(94.7) 10(5.3)$ & $85(94.4) 5(5.6)$ & $94(94.9) 5(5.1)$ & 0.87 \\
\hline
\end{tabular}


[31] showed that ivermectin, as an adjunct to other prospective drugs, reduced mortality and resulted in clinical improvement. Furthermore Okumuş et al. also found ivermectin to be beneficial as an add-on therapy [32]. However, the use of other drugs can interfere with ivermectin metabolism. Antiretrovirals such as lopinavir/ritonavir and darunavir/cobicistat which inhibit
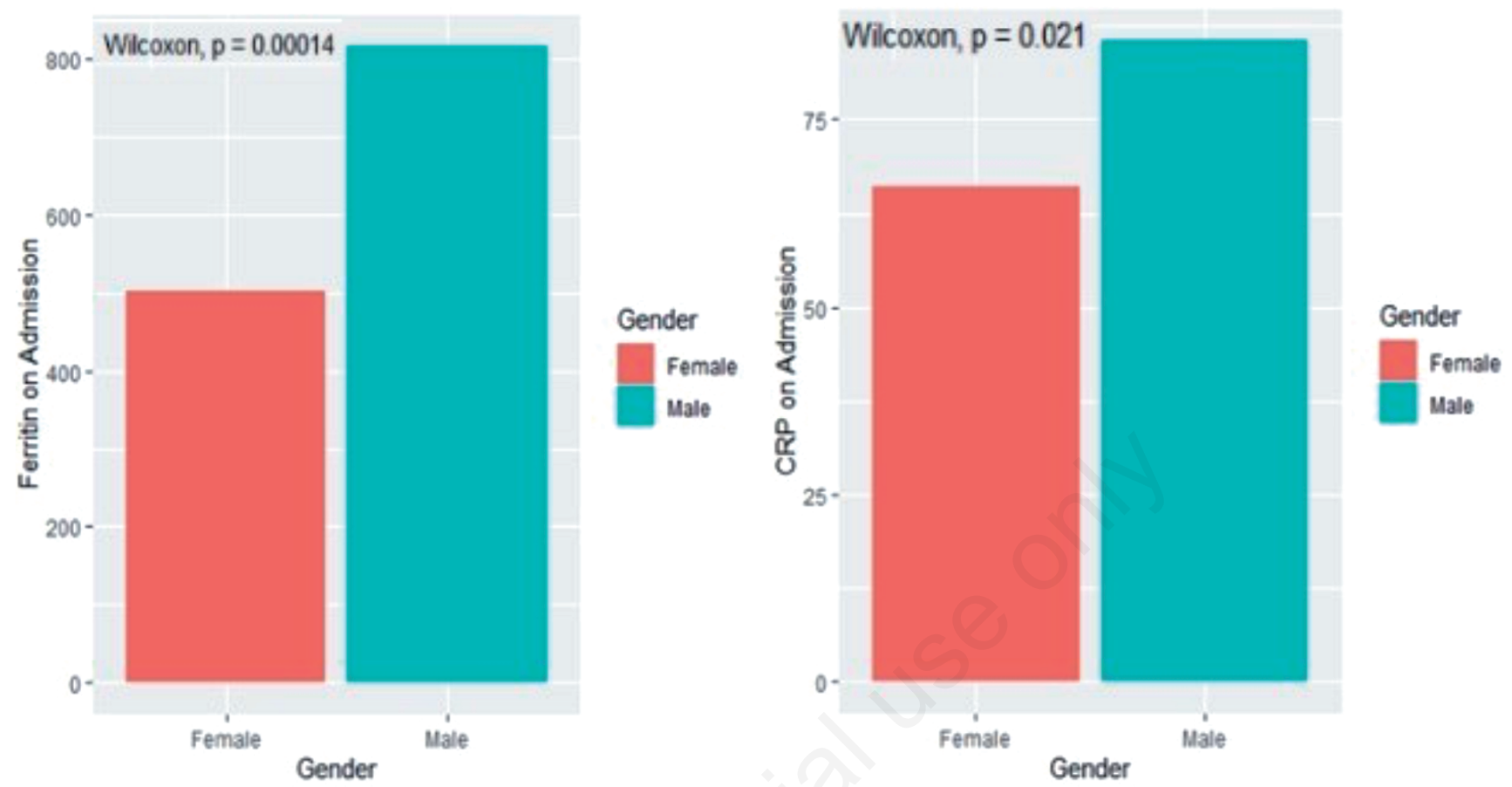

Figure 1. Gender base difference of serum ferritin and C-reactive protein levels on admission.

Table 3. Gender base difference on C-reactive protein, ferritin level, length of hospitalization and outcome in both study and control group.

\begin{tabular}{|c|c|c|c|c|c|c|c|}
\hline & Study & group & Control & group & Tot: & & p-value \\
\hline & $\begin{array}{l}\text { Female } \\
(\mathrm{n}=28)\end{array}$ & $\begin{array}{c}\text { Male } \\
(n=62)\end{array}$ & $\begin{array}{l}\text { Female } \\
(\mathrm{n}=34)\end{array}$ & $\begin{array}{c}\text { Male } \\
(\mathrm{n}=64)\end{array}$ & $\begin{array}{l}\text { Female } \\
(\mathrm{n}=62)\end{array}$ & $\begin{array}{c}\text { Male } \\
(\mathrm{n}=126)\end{array}$ & $(\mathrm{OR})$ \\
\hline CRP on admissio. & & & & & & & \\
\hline Mean (SD) & $74.9(57.8)$ & $86.2(59.5)$ & $58.2(65.8)$ & $84.9(63.6)$ & $66.0(62.2)$ & $85.5(61.4)$ & 0.021 \\
\hline Median (min, max) & $61.2(3.58,249)$ & $76.4(5.06,200)$ & $40.4(0.400,306)$ & $75.4(0.26,264)$ & $48.0(0.400,306)$ & $75.9(0.260,264)$ & \\
\hline CRP on discharge & & & & & & & \\
\hline Mean (SD) & $29.0(24.4)$ & $26.9(30.0)$ & $26.8(30.0)$ & $35.5(40.4)$ & $27.7(27.5)$ & $31.1(35.6)$ & 0.866 \\
\hline Median (min, max) & $23.3(1.16,88.1)$ & $20.4(0.300,187)$ & $18.7(0.140,122)$ & $18.0(0.450,166)$ & $19.2(0.140,122)$ & $18.8(0.300,187)$ & \\
\hline Ferritin on admis & & & & & & & \\
\hline Mean (SD) & $589(686)$ & $753(845)$ & $426(540)$ & $880(648)$ & $502(612)$ & $815(755)$ & \\
\hline Median (min, max) & $401(44.6,3380)$ & $530(49.1,5090)$ & $262(3.10,2590)$ & $615(28.6,2970)$ & $301(3.10,3380)$ & $555(28.6,5090)$ & $<0.001$ \\
\hline Ferritin on disch & & & & & & & \\
\hline Mean (SD) & $571(677)$ & $749(671)$ & $460(469)$ & 1050 (649) & $523(591)$ & $879(675)$ & $<0.001$ \\
\hline Median (min, max) & $435(65.1,3270)$ & $597(30.3,3630)$ & $271(29.6,1720)$ & $1040(140,2930)$ & $304(29.6,3270)$ & $708(30.3,3630)$ & \\
\hline Length of hospit & & & & & & & \\
\hline Mean (SD) & $4.89(2.08)$ & $4.85(2.99)$ & $3.88(1.65)$ & $4.83(2.56)$ & $4.34(1.91)$ & $4.84(2.77)$ & 0.40 \\
\hline Median (min, max) & $4.50(2.00,10.0)$ & $5.00(1.00,18.0)$ & $4.00(1.00,8.00)$ & $4.00(1.00,15.0)$ & $4.00(1.00,10.0)$ & $4.00(1.00,18.0)$ & \\
\hline Outcome & & & & & & & \\
\hline Discharged & $26.0(92.9 \%)$ & $59.0(95.2 \%)$ & $33.0(97.1 \%)$ & $60.0(93.8 \%)$ & $59.0(95.2 \%)$ & $119(94.4 \%)$ & 1.00 \\
\hline Expired & $2.00(7.1 \%)$ & $3.00(4.8 \%)$ & $1.00(2.9 \%)$ & $4.00(6.3 \%)$ & $3.00(4.8 \%)$ & $7.0(5.6 \%)$ & \\
\hline
\end{tabular}

CRP, C-reactive protein; SD, standard deviation. 
cytochrome P450 3A4 - metabolizes ivermectin. Ritonavir and cobicistat also inhibit the P-glycoprotein which usually takes ivermectin out of the CNS, thereby facilitating toxic doses $[33,34]$.

Differences in inflammatory markers in the two genders were noted. Higher ferritin and CRP levels in men compared to women might point to a worse prognosis in males. Similar results have been noted in other studies $[35,36]$. These two studies go on to propose that testosterone has an immunosuppressive effect which leads to greater susceptibility. Indeed, a reduction in testosterone has been linked to greater susceptibility such as in men of old age [37]. A study from China in 2020 concluded that males are 'at risk for worse outcomes and death' independent of age [38]. A study from Italy corroborates this notion by reporting an increased mortality rate in men [39]. However, the same study did also report a higher incidence in women, which is in contrast to studies suggesting the opposite [40]. In a large US cohort where comorbidities were matched, males were observed to be at risk for worse outcomes [40]. Other possible explanations have been offered - one of which is a higher ACE2 concentration in men [41]. Attitudes have also been shown to differ amongst men and women [42]. Our study though does not provide evidence for a higher mortality or longer stay in males as explained below. We do encourage researchers to look into these differences.

Our study supports the hypothesis that increased age is related to higher mortality in COVID-19 patients as in other studies [43].Ferritin has been shown to be related to fatal outcomes in COVID-19 due to its involvement with the cytokine storm $[44,45]$. Our results show significantly less ferritin levels were observed in the ivermectin arm, which would suggest ivermectin did positively affect in the therapy of SARS-CoV-2. It might still be possible that the effect of ivermectin might not translate into improvement in length of hospital stay. This could explain why the difference in inflammatory marker levels did not seem to affect length of stay or mortality in the two genders as well. Literature on the effect of ivermectin on these parameters is variable $[18,46]$.

We recommend that high-powered randomized controlled trials to determine the role of ivermectin in the treatment of SARSCoV-2.

\section{Limitations}

Retrospective cohorts are prone to informational bias. Potential differences between groups might not be detected due to smaller sample size. Other treatment for COVID-19 like the use of steroids and remdesivir, was more pronounced in the study group. The dosage of ivermectin across all subjects in the study was not standardized.

\section{Conclusions}

This study does not provide evidence for the use of ivermectin in the treatment of COVID-19 in severe and non-severe hospitalized patients. Furthermore, there was no significant difference seen in any gender with respect to effect of ivermectin. Large scale RCTs are required to evaluate the role of ivermectin in the COVID-19 disease.

\section{References}

1. World Health Organization. Weekly epidemiological update on COVID-19. Available from: https://www.who.int/publications/m/item/weekly-epidemiological-update-on-covid-1918-may-2021

2. Government of Pakistan. COVID-19 Health Advisory Platform by Ministry of National Health Services Regulations \& Coordination 2021. Available from: https://covid.gov.pk/ stats/pakistan

3. Pan H, Peto R, Henao-Restrepo AM, et al. Repurposed antiviral drugs for Covid-19 - Interim WHO solidarity trial results. N Engl J Med 2021;38):497-511.

4. Hermine O, Mariette X, Tharaux PL, et al. Effect of tocilizumab vs usual care in adults hospitalized with COVID-19 and moderate or severe pneumonia: A randomized clinical trial. JAMA Intern Med 2021;181:32-40.

5. Agarwal A, Mukherjee A, Kumar G, et al. Convalescent plasma in the management of moderate covid-19 in adults in India: open label phase II multicentre randomised controlled trial (PLACID Trial). BMJ 2020;371:m3939.

6. Tufan A, Avanoğlu Güler A, Matucci-Cerinic M. COVID-19, immune system response, hyperinflammation and repurposing antirheumatic drugs. Turk J Med Sci 2020;50:620-32.

7. Horby P, Lim WS, Emberson JR, et al. Dexamethasone in hospitalized patients with Covid-19. N Engl J Med 2021;384:693-704.

8. Kalil AC, Patterson TF, Mehta AK, et al. Baricitinib plus remdesivir for hospitalized adults with Covid-19. N Engl J Med 2021;384:795-807.

9. Yang SNY, Atkinson SC, Wang C, et al. The broad spectrum antiviral ivermectin targets the host nuclear transport importin $\alpha / \beta 1$ heterodimer. Antiviral Res 2020;177:104760.

10. Wang X, Lv C, Ji X, et al. Ivermectin treatment inhibits the replication of Porcine circovirus 2 (PCV2) in vitro and mitigates the impact of viral infection in piglets. Virus Res 2019;263:80-6.

11. Priel A, Silberberg SD. Mechanism of ivermectin facilitation of human P2X4 receptor channels. J Gen Physiol 2004;123:281-93.

12. Zhang X, Song Y, Ci X, et al. Ivermectin inhibits LPS-induced production of inflammatory cytokines and improves LPSinduced survival in mice. Inflamm Res 2008;57):524-9.

13. Caly L, Druce JD, Catton MG, et al. The FDA-approved drug ivermectin inhibits the replication of SARS-CoV-2 in vitro. Antiviral Res 2020;178:104787.

14. Eurekalert [Internet]. Latest peer-reviewed research: Immediate global ivermectin use will end COVID-19 pandemic (2021, May 7). Last accessed on 19th July 2021. https://eurekalert.org/pub_ releases/2021-05/fccc-lpr050621.php

15. No authors listed. Update to living WHO guideline on drugs for covid-19. BMJ 2021;372:n860.

16. Rajter JC, Sherman MS, Fatteh N, et al. Use of ivermectin is associated with lower mortality in hospitalized patients with coronavirus disease 2019: the ivermectin in COVID nineteen study. Chest 2021;159:85-92.

17. Chaccour C, Casellas A, Blanco-Di Matteo A, et al. The effect of early treatment with ivermectin on viral load, symptoms and humoral response in patients with non-severe COVID-19: A pilot, double-blind, placebo-controlled, randomized clinical trial. EClinicalMedicine 2021;32:100720.

18. López-Medina E, López P, Hurtado IC, et al. Effect of ivermectin on time to resolution of symptoms among adults with 
mild COVID-19: A randomized clinical trial. JAMA 2021;325: 1426-35.

19. Ahmed Elgazzar BH, Shaimaa Abo Youssef, Basma Hany, et al. Efficacy and safety of ivermectin for treatment and prophylaxis of COVID-19 pandemic. Research Square 2021 (Withdrawn). Available from: https://assets.researchsquare.com/files/rs100956/v2/c11416a2-d0bd-494f-abc8-3cbf8c605b10.pdf?c= 1631861037

20. Morteza Shakhsi N, Nematollah G, Peyman N, et al. Ivermectin as an adjunct treatment for hospitalized adult COVID-19 patients: A randomized multi-center clinical trial. Research Square 2021. Available from: https://www.researchsquare.com/article/rs-109670/v1

21. Ravikirti, Roy R, Pattadar C, et al. Ivermectin as a potential treatment for mild to moderate COVID-19 - A double blind randomized placebo-controlled trial. medRxiv 2021:2021.01. 05.21249310 .

22. Gorial FI, Mashhadani S, Sayaly HM, et al. Effectiveness of ivermectin as add-on therapy in COVID-19 management (Pilot Trial). medRxiv 2020:2020.07.07.20145979.

23. Chachar AZK, Kahn KA, Asif M, et al. Effectiveness of ivermectin in SARS-CoV-2/COVID-19 patients. Int J Sci 2020;9:31-5.

24. National Institutes of Health. Table 2c. Ivermectin: Selected Clinical Data: NIH; 2021. Updated 11 Februrary 2021. Available from: https://www.covid19treatmentguidelines. nih.gov/tables/table-2c/

25. Camprubí D, Almuedo-Riera A, Martí-Soler H, et al. Lack of efficacy of standard doses of ivermectin in severe COVID-19 patients. PLoS One 2020;15:e0242184.

26. Wimmersberger D, Coulibaly JT, Schulz JD, et al. Efficacy and safety of ivermectin against trichuris trichiura in preschoolaged and school-aged children: A randomized controlled dosefinding trial. Clin Infect Dis 2018;67:1247-55.

27. Smit MR, Ochomo EO, Aljayyoussi G, et al. Human direct skin feeding versus membrane feeding to assess the mosquitocidal efficacy of high-dose ivermectin (IVERMAL Trial). Clin Infect Dis 2019;69:1112-9.

28. Buonfrate D, Salas-Coronas J, Muñoz J, et al. Multiple-dose versus single-dose ivermectin for Strongyloides stercoralis infection (Strong Treat 1 to 4): a multicentre, open-label, phase 3 , randomised controlled superiority trial. Lancet Infect Dis 2019;19):1181-90.

29. European Medicine Agency. EMA advises against use of ivermectin for the prevention or treatment COVID-19 outside randomised clinical trials. 2021, March 22. Last accessed on 19th July 2021. Available from: https:/www.ema.europa.eu/en/ news/ema-advises-against-use-ivermectin-prevention-treatment-covid-19-outside-randomised-clinical-trials

30. National Institutes of Health. COVID-19 treatment. Ivermectin. Available from: www.covid19treatmentguidelines.nih.gov/antiviral-therapy/ivermectin/
31. Padhy BM, Mohanty RR, Das S, Meher BR. Therapeutic potential of ivermectin as add on treatment in COVID 19: A systematic review and meta-analysis. J Pharm Pharm Sci 2020;23:462-9.

32. Okumuş N, Demirtürk N, Çetinkaya RA, et al. Evaluation of the effectiveness and safety of adding ivermectin to treatment in severe COVID-19 patients. BMC Infect Dis 2021;21:411.

33. Lepist EI, Phan TK, Roy A, et al. Cobicistat boosts the intestinal absorption of transport substrates, including HIV protease inhibitors and GS-7340, in vitro. Antimicrob Agents Chemother 2012;56:5409-13.

34. Drewe J, Gutmann H, Fricker G, et al. HIV protease inhibitor ritonavir: a more potent inhibitor of P-glycoprotein than the cyclosporine analog SDZ PSC 833. Biochem Pharmacol 1999;57:1147-52.

35. Hampton T. Insight on sex-based immunity differences, with COVID-19 implications. JAMA 2020;324:1274.

36. Haitao T, Vermunt JV, Abeykoon J, et al. COVID-19 and sex differences: Mechanisms and biomarkers. Mayo Clin Proc 2020;95:2189-203.

37. Takahashi T, Iwasaki A. Sex differences in immune responses. Science 2021;371:347348.

38. Jin JM, Bai P, He W, et al. Gender differences in patients with COVID-19: Focus on severity and mortality. Front Public Health 2020;8:152.

39. Mangia C, Russo A, Civitelli S, et al. [Sex/gender differences in COVID-19 lethality: what the data say, and do not say].[Article in Italian]. Epidemiol Prev 2020;44:400-6.

40. Vahidy FS, Pan AP, Ahnstedt H, et al. Sex differences in susceptibility, severity, and outcomes of coronavirus disease 2019: Cross-sectional analysis from a diverse US metropolitan area. PLoS One 2021;16:e0245556.

41. Oudit GY, Pfeffer MA. Plasma angiotensin-converting enzyme 2: novel biomarker in heart failure with implications for COVID-19. Eur Heart J 2020;41:1818-20.

42. Galasso V, Pons V, Profeta P, et al. Gender differences in COVID-19 attitudes and behavior: Panel evidence from eight countries. Proc Natl Acad Sci USA 2020;117:27285-91.

43. Zhou F, Yu T, Du R, et al. Clinical course and risk factors for mortality of adult inpatients with COVID-19 in Wuhan, China: a retrospective cohort study. Lancet 2020;395:1054-62.

44. Zhou B, She J, Wang Y, et al. Utility of ferritin, procalcitonin, and C-reactive protein in severe patients with 2019 novel coronavirus disease. Research Square 2020. Available from: https://www.researchsquare.com/article/rs-18079/v1

45. Abbaspour N, Hurrell R, Kelishadi R. Review on iron and its importance for human health. J Res Med Sci 2014;19:164.

46. Ahmed S, Karim MM, Ross AG, et al. A five-day course of ivermectin for the treatment of COVID-19 may reduce the duration of illness. Int J Infect Dis 2021;103:214-6. 